Indonesian Journal of Cardiology

Indonesian J Cardiol 2020:41:206-2/2

pISSN: 0I26-3773 / elSSN: 2620-4762

doi: $10.30701 /$ ijc. 1069

\title{
A Significant Addition of Left Ventricular Fractional Shortening to Ejection Fraction Correlated with Global Longitudinal Strain Value in Predicting Major Acute Cardiovascular Event in Patients with Acute Coronary Syndrome
}

' Faculty of Medicine, Universitas Sumatera Utara

${ }^{2}$ Department of Cardiology and Vascular Medicine, Faculty of Medicine, Universitas Sumatera Utara, Medan, Indonesia

Correspondence:

Bertha Gabriela Napitupulu

Faculty of Medicine, Universitas

Sumatera Utara

Email: berthanapitupulu@gmail.com

\author{
Bertha Gabriela Napitupulu', Harris Hasan², Nizam Z Akbar², \\ Andre Pasha Ketaren², Zainal Safri ${ }^{2}$, Ali Nafiah Nasution ${ }^{2}$
}

\begin{abstract}
Background: Global longitudinal strain (GLS) was a proven predictor of systolic function improvement and myocardial remodeling after acute coronary syndrome (ACS) for a residual left ventricular function defined their prognosis. However, not all echocardiography devices are equipped by speckle tracking (STE) as compare to the availability of M-mode modality which capable on assessing fractional shortening (FS) instead.

Methods: This study evaluated clinical and echocardiography parameters on myocardial infarction (MI) and non-MI ACS patients. Clinical outcome was defined as composite major acute cardiovascular event (MACE) on 6 months of follow up.

Results: Over 145 patients, GLS>-9.4\% was found to be an independent predictor of MACE despite of troponin, age, ejection fraction (EF), prior reperfusion and infarct location [(HR $5.89(\mathrm{I} .82-\mathrm{I} 6.5 \mathrm{I})]$. There is negative correlation between FS and GLS (Spearman $r-0.717 ; p<0.0 \mathrm{I})$. By using logistic regression analyses, it was found that the addition of $\mathrm{FS}<25 \%$ to biplane Simpson $E F<50 \%$ could be useful to rule in the presence of GLS $>$ 9.4\% (AUC 0.83I).

Conclusion: GLS had a prognostic value in patients with ACS. Left ventricular conventional M-mode FS in addition to Simpson EF were well correlated with GLS as well they can be considered as an alternative in predicting the incident of MACE in patient with ACS.
\end{abstract}

(Indonesian J Cardiol. 2020;41:206-2I2)

Keywords: global longitudinal strain, prognostic, fractional shortening, acute coronary syndrome 


\section{Introduction}

A cute Coronary Syndrome (ACS) was a term used to describe symptoms caused by myocardium ischemia that occurs suddenly, decreasing perfusion to the myocardium. ${ }^{1}$ In Indonesia, Jakarta Acute Coronary Syndrome (JAC) annual registry analysis of the National Heart Center Emergency Department of Harapan Kita HospitalIndonesia (PJNHK) showed there were totally 2103 ACS patients, of which the majority of STEMI patients (59\%) did not receive acute reperfusion therapy and $52 \%$ of patients were inter-hospitalization patients where mortality rates during the hospitalization period of STEMI patients who did not receive higher reperfusion therapy compared to STEMI patients who received both fibrinolysis and primary PCI $(13.3 \%$ vs. $6.2 \%$ vs. $5.3 \%){ }^{2}$ Each year, 1.5 million people in America were diagnosed with myocardial infarction so that the treatment given to post-ACS patients was no longer just to improve the symptoms and functional performance of patients, but to prevent the progressivism of systolic dysfunction from the left ventricle. Dysfunction of the left ventricular myocardium was started from the distal myocardial segment and gradually moved toward the basal segment, so myocardial function could be assessed regionally or globally in order to get the effect of this regional ischemia on global systolic function by the mean of echocardiography in the form of ejection fraction, fractional shortening (FS) value, or global longitudinal strain (GLS) value. ${ }^{3-5}$ In sub-acute phase (above 48 hours) of ischemia and myocardial infarction, echocardiography is used for diagnosis of heart failure and assessment of the severity of clinical systolic and diastolic heart failure through the use of ejection fraction parameters and strain. ${ }^{4}$ It turns out that in ACS patients, GLS found to be a significant index to assess the long-term prognosis i.e. as a predictor of systolic function improvement and reverse remodeling events within 6 months. ${ }^{6,7}$ Fractional Shortening (FS) measurement with $\mathrm{M}$-mode modality have a similar concept with GLS calculations which namely utilizing high temporal resolution to assess the dimensions of the left ventricle, yet it is just that FS assesses the length of longitudinal fibers in two segments along the cursor pieces. ${ }^{5}$ Some studies found a significant strong correlation between GLS and FS values, only that this study was not focused on the ACS patient population. ${ }^{8,9}$ Studies on GLS significance has only grown in the last 5 years so when compared to speckle tracking, the likelihood of M-mode modality availability would be higher than speckle tracking echo especially in centers with standard or limited diagnostic resources. Therefore, a study would be needed to obtain the correlation of a simple conventional FS using M-Mode modality with a GLS, a proven systolic function parameter which strongly associated with ACS prognosis

\section{Methods}

This prospective cohort study assessed the correlation between conventional M-Mode Fractional Shortening (FS) and speckle tracking Global Longitudinal Strain (GLS) of the left ventricle in acute coronary syndrome patients Figure 1 using the GE Vivid S6 tool with a 3.2 $\mathrm{MHz}$ frequency sector heart probe. There were a total of 145 firstly diagnosed ACS patients with already a stable clinical condition when echocardiography were performed after minimally 72 hours of their onset. We included only patients with sinus rhythm and those who

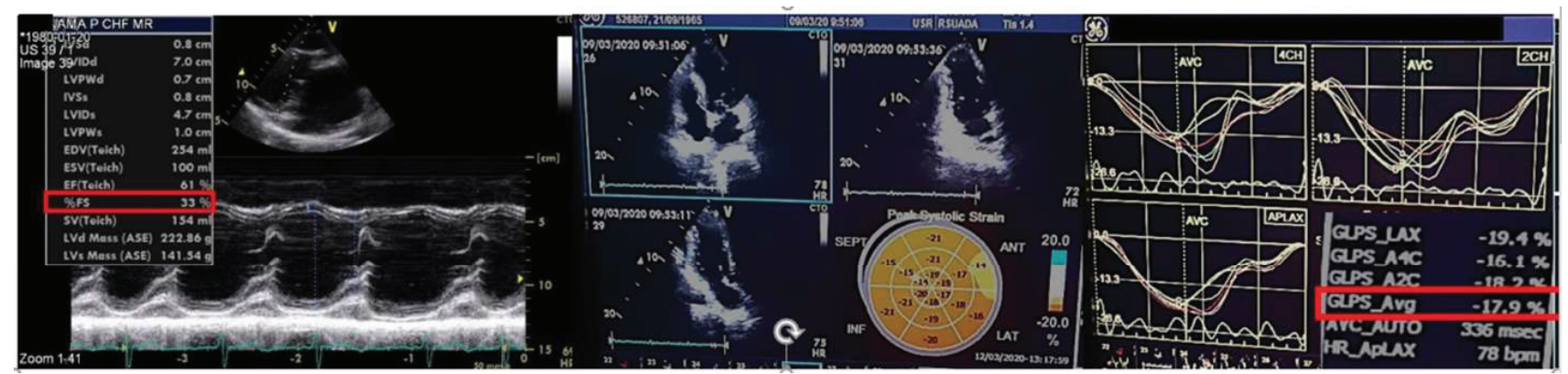

Figure 1. (1A). Left ventricular M-Mode Fractional Shorterning on view 2D PLAX with cursor perpendicularly placed over IVS and parallel to the tip of mitral leaflet. (1B) Average GLS from 17 segments using speckle tracking modality. 
weren't meet the criteria of cardiomyopathy dilation or hypertrophic cardiomyopathy, patients with primary valve abnormalities and or with cardiac congenital abnormalities resulting in hemodynamic changes or patients with previous heart surgery, implanted intra cardiac device or pacemakers and or prosthesis. As a secondary outcome, survival analysis would be conducted to predict MACE (Major adverse cardiovascular events) within 6 months based on a significant cut off of GLS values. Then, sample would be divided based on that GLS value and the analysis continued to assess the correlation and comparison of left ventricular echocardiography indices in terms of distinguishing the group from the GLS value that obtained. MACE were assessed where it was a composite of the incidence of death or rehospitalization due to acute heart failure, myocardial infarction and arrhythmia assessed in 6 months. P value $<0.05$ were found to be significant.

\section{Result}

There were 29 (20\%) Unstable Angina Pectoris patients, $53(36.6 \%)$ NSTEMI patients, and 63 (43.4\%) STEMI patients with 22 patients $(15.2 \%)$ of inferior region infarction and 41 patients $(28.3 \%)$ with the anterior region involved. The mean age were $55.67 \pm 9.8$ years with 53 people $(86.9 \%)$ and mean Simpson EF of $45.03 \pm 11.52$, Teich EF $49.23 \pm 10.56$ and FS 28.9 \pm 10.02 . ACS patients in this study had decreased GLS scores with a median value $(-11.1)$ and the worst value was $(-3.2)$ with $35.9 \%$ had already been revascularized when echocardiography performed (Table 1). Patients included in the study had an average pain onset of $59 \pm 22.45$ hours. Patients who had not been revascularized were mostly patients without any significant signs of ischemia or those who came after the onset of revascularization targets or multivessel coronary patients planned to have coronary bypass graft surgery so that it was still conservatively managed not only when the echocardiography was performed but also until within 6 months of follow up.

There were no differences found in GLS value based on the infarcted area on STEMI, revascularization history, and based on the EF value. There were five routine echocardiographic findings that have a good correlation (Spearman rho) with GLS as Simpson EF ( $\mathrm{r}$ 0.695; p <0.01), Teich EF (r 0.654; p <0.01), LVESD ( $r$
Table 1. Baseline Characteristics

\begin{tabular}{lc}
\hline \multicolumn{1}{c}{ Parameter } & Value $(\mathbf{n}=\mathbf{1 4 5})$ \\
\hline Age, years (mean+SD) & $55.28 \pm 8.37$ \\
Male, $\mathrm{n}(\%)$ & $127(87.6)$ \\
Infarct, n (\%) & $116(80)$ \\
Pain onset, hours (mean+SD) & $59 \pm 22.45$ \\
Revascularized, n (\%) & $52(35.9)$ \\
LVEDV, mL (min-max) & $101.28 \pm 31.48$ \\
LVESV, mL (min-max) & $57.72 \pm 27.81$ \\
LVEDD, mm (min-max) & $55.7 \pm 8.9$ \\
LVESD, mm (min-max) & $40.44 \pm 9.27$ \\
Teich EF, \% (mean+SD) & $49.23 \pm 10.56$ \\
Simpson EF, \% (mean+SD) & $45.03 \pm 11.52$ \\
FS, \% (mean+SD) & $28.9 \pm 10.02$ \\
GLS, \% (min-max) & $(-23.5)-(-3.2)$ \\
E/e', mm (min-max) & $12.01 \pm 4.15$ \\
Medication & \\
Anticoagulant, n (\%) & $145(100)$ \\
Betablocker, n (\%) & $91(62.75)$ \\
ACE/ARB, n (\%) & $145(100)$ \\
MRA, n (\%) & $50(34.48)$ \\
Statin, n (\%) & $145(100)$ \\
Nitrate, n (\%) & $100(68.9)$ \\
Diuretics, n (\%) & $60(41.37)$ \\
\hline
\end{tabular}

0.317; $\mathrm{p}<0.01$ ), LVESV ( $\mathrm{r} 0.510 ; \mathrm{p}<0.01)$, FS value ( $\mathrm{r}$ $0.717, \mathrm{p}<0.01)$, and the E / e 'value (r 0.421; p <0.01). There was a significant negative correlation between the FS and GLS values in the ACS patients who were the sample of this study $(\mathrm{r}-0.717 ; \mathrm{p}<0.01)$, with a decrease in the FS value followed by an increasingly positive GLS value (Figure 2).

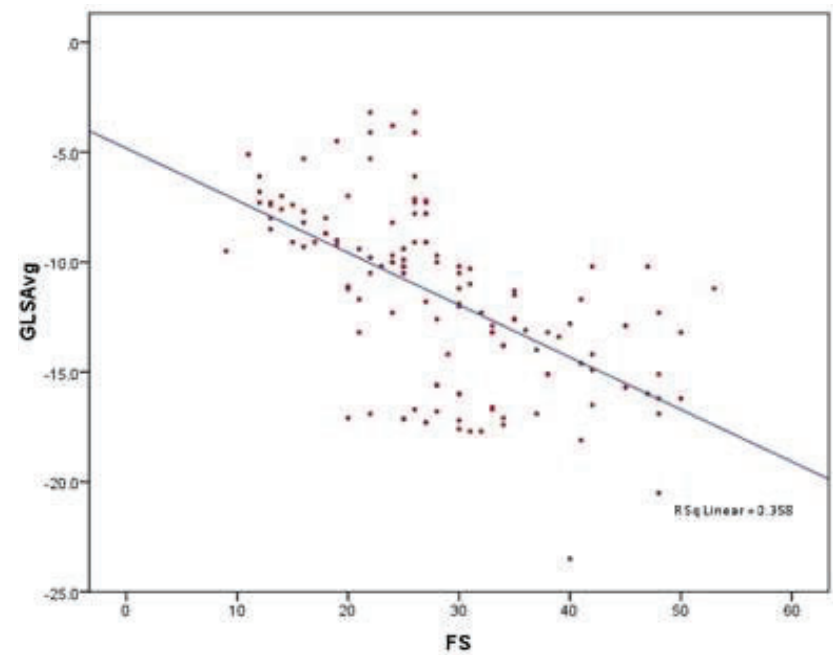

Figure 2. Scatter plot of significant correlation between average Global Longitudinal Strain (GLS\%) and Fractional Shortening (FS\%). 
There were 35 people who had MACE in the sample. By using Cox regression analysis, incidence was found a different between groups with GLS <-9.4\% with GLS> $-9.4 \%$ with HR 5.89 (1.82-16.51) as depicted on the Kaplan-Meier curve below, where 27 samples with GLS> $-9.4 \%$ suffered MACE within 6 months $(-9.4 \%$ $(-3.2 \%))$ (Figure 3)

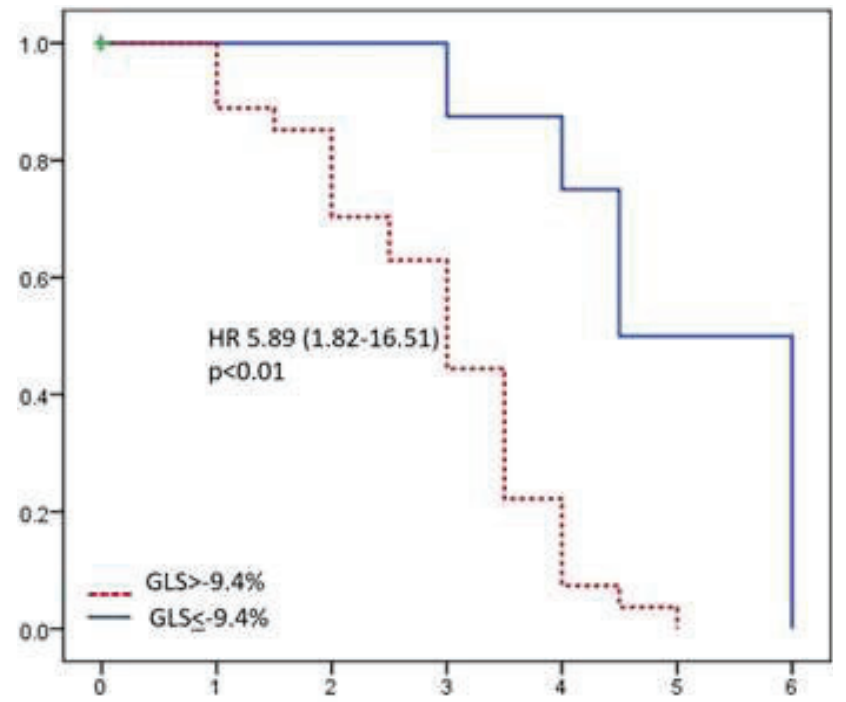

Figure 3. Kaplan Meier curve on survival analysis within 6 months follow up.

We decided to find a cut-off point of FS value that could differ GLS values> $-9.4 \%$. From the ROC (Receiver Operating Characteristic) curve, it was found that the FS value could significantly be used as a parameter to distinguish the GLS value> $-9.4 \%$ from the better GLS value namely GLS $<-9.4 \%$, with a very good discrimination ability (AUC of 0.886 (confidence interval $0.833-0.939$ ) with a $\mathrm{p}$ value $<0.01$ ) with FS cutoff value is $25 \%$.

Other echocardiographic parameters that correlated statistically with GLS were multivariate analyzed using backward binary logistic regression to have the powered significant parameter that predict GLS. The combined use of Simpson's FS and EF parameters can represent the GLS value with a good power rating of AUC 0.831 $(0.755$ - 0.906). The addition of FS to Simpson EF was proved to increase the strength of the significant relationship in predicting GLS values (Table 2) rather than he use of Simpson EF alone (Model 2), or FS alone (Model 3), so that the addition of FS to the Simpson EF did not decrease the strength of the Simpson EF value
Table 2. Comparison between Simpson EF and FS to differ GLS >-9.4\%

\begin{tabular}{llcc}
\hline Model & Parameter & Adjusted $\mathbf{R}^{2}$ & $\begin{array}{c}\text { AUC predicted } \\
\text { probability }\end{array}$ \\
\hline 1 & FS+Simpson EF & 0.417 & 0.831 \\
& & & $(0.755-0.906)$ \\
2 & Simpson EF & 0.199 & 0.675 \\
3 & FS & 0.321 & $(0.587-0.763)$ \\
& & & 0.763 \\
& & & $(0.670-0.855)$ \\
\hline
\end{tabular}

in predicting GLS in ACS patients (Table 2). Therefore, we compared the sensitivity and specificity values of FS, Simpson EF, and Teich EF in differentiating GLS>$9.4 \%$ (Table 3 )

Table 3. Comparison of Sensitivity and Specificity between Simpson EF, Teich, FS and GLS

\begin{tabular}{llccc}
\hline \multicolumn{1}{c}{ Parameter } & $>-9.4 \%$ & $<-9.4 \%$ & Sensitivity* $^{*}$ & Sensitivity* $^{*}$ \\
\hline FS (\%) & & & & \\
$<25$ & 29 & 17 & $69 \%$ & $83.5 \%$ \\
$>25$ & 13 & 86 & & \\
Simpson EF (\%) & & & & \\
$<50$ & 40 & 62 & $95.2 \%$ & $60.2 \%$ \\
$>50$ & 2 & 41 & & \\
Teich EF (\%) & & & & \\
$<50$ & 28 & 29 & $66.7 \%$ & $74 \%$ \\
$>50$ & 14 & 74 & & \\
Total & 42 & 103 & & \\
\hline
\end{tabular}

In its ability to detect GLS> $-9.4 \%$, the FS value $<25 \%$ has good specificity compared to the Simpson EF value $<50 \%$ ( $83.5 \%$ vs $60.2 \%)$ although the sensitivity were not so good when compared to Teich EF and Simpson EF (Table 3). It appears that the Simpson EF value $<50 \%$ has a high false positive value, whereas at the same time, an FS value> $25 \%$ with a higher specificity to find patients with GLS that have a better prognosis (GLS <- 9.4\%)

\section{Discussion}

The use of echocardiography in the incidence of acute coronary syndrome is recommended as a standard diagnostic tool in detecting systolic dysfunction of the left ventricular myocardium and degree of ischemia. 10 GLS was not only found to be more significant and sensitive parameters than ejection fractions to the evaluate left ventricular abnormalities but also found as reliable indicators in terms of prognosis. GLS 
scores with a cut off of less than $-9.4 \%$ are associated with worsening incidence of heart failure in coronary heart disease patients who include post-acute coronary syndrome patients. ${ }^{11}$

In the early phases of persistent ischemia and myocardial infarction, necrosis and ischemic expansion occurred for less than 72 hours, followed by neurohormonal system compensation which was originally an adaptive response to increase cardiac mass, so that in the initial phase for some myocardial infarctions with an area of less than $25 \%$, ejection fraction was still and heart dimensions would not change from baseline. This process would result in a settled contractility and the dimensions of the left ventricle for at least the first 1 week. ${ }^{3}$ Our study included samples that had been performed echocardiography 72 hours from the pain onset. The myocardial remodeling process continued to the healing phase of infarction and granulation which would result in myocardial hypertrophy and structural changes in an effort to distribute increased wall stress. This phase mainly begun in one week after myocardial infarction. And after that it would go into the advanced remodeling phase where there would be progressive dilation due to the eccentric hypertrophy process and lengthening of the non-infarction myocardial segment in up to 6 months post-infarction. ${ }^{3}$ In the ischaemic and infarcted myocardium area, there would be irreversible damage and loss of synchronization with other myocardial areas followed by the commencement of compensation of other areas especially in the subendocardial area of longitudinal fibers. GLS assessed the movement specifically of those longitudinal fibers, and research by $\mathrm{Hu}$ also found that GLS scores with a significant cut off of $-10.4 \%$ were predictors of EF improvement of at least $5 \%$ accompanied by a $20 \%$ increase in LVEDV assessed at the end of the sixth month of acute coronary syndrome, and $\mathrm{Hu}$ found that GLS was much more significant than ejection fraction or WMSI fractions. The movement of longitudinal fibers plays more of a role in ejection fractions, while circumference fibers play a role in the shape and size of the enviable ventricle, so the preservation of longitudinal fibers would determine the likelihood of increased ejection fraction, while the damage of circumference fibers will accelerate the remodeling of the left ventricle. ${ }^{6}$ In addition, study found that GLS value $>-15 \%$ is related to the incidence of left ventricular dilation in the $3^{\text {td }}$ and $6^{\text {th }}$ months in post-myocardial infarction patients that PCI had performed. ${ }^{12}$

FS values are strongly influenced by chamber diameter from M-Mode measurements on PLAX views that measure the diameter of the left ventricular anteroposterior. The mean FS in this study was $28.9+10.02$ and measured in conjunction with Teich EF. This measurement was known as endocardial FS or conventional FS which turns out to increase in value with age, with the normal value in research in Korea being 30-40\%. ${ }^{13-14}$ In 97 young patients who performed echocardiography 6-12 weeks after myocardial infarction, after being prospectively followed against MACE post-infarction, there was a difference in FS value between patients with non-fatal reinfarction and patients with death $(30 \%$ vs. $24 \% ; \mathrm{p}<0.05)$ with an average follow up duration of 6 years. FS scores were also significantly found to be predictors of death due to cardiovascular events, along with ejection fraction, LVEDD, and LVESD. That study also found that EPSS (E point septal separation) is not a prognostic indicator and proved LVESD and FS as significant parameters in determining the long-term mortality of post-infarction patients, along with the current heart rate of admission, ejection fraction, and multivesel conditions with an average of FS $18 \pm 4 \% .^{15}$

Huang et al study found a strong correlation between FS and GLS with a correlation strength of 0.86 . The difference was that the FS value found from Huang study did not use conventional FS with M-Mode from PLAX view but from the combination of the total tracing value of the length of the left ventricular wall from the baseapex with MAPSE taken from the $\mathrm{A} 4 \mathrm{CH}$ view. That FS measurement had better correlation values, only that the measurements was more complicated than conventional FS measurements from M-mode done in our study. Conventional FS measurement was easier because it does not depend on the image quality and angle of M-mode cursor retrieval. ${ }^{8}$ Research conducted by Adachi et al with a slightly lower mean EF also found that there was an excellent correlation between GLS and FS with a correlation coefficient of 0.93 where their study samples were patients with an mean EF $38.5+15 \%$, mean GLS $-15.5+6.1 \%$, and mean FS value $14.7+5.5 \%$. From this study obtained GLS value $>-9 \%$ has $100 \%$ sensitivity and $99 \%$ specificity to differ $\mathrm{EF}<35 \% .^{9}$ In ACS patients with reduced EF, GLS capabilities would be more 
beneficial in terms of prognostic and predict mortality. This is seen from a linear relationship with GLS values $-11 \%$ associated with an EF range value of $35 \%$ with or without wall motion abnormalities. 16 Apical's role in producing a contraction would greatly affect ejection fraction due to the portion of myocardial strength and torsion capable of altering left ventricular dimensions of the heart chamber at systolic time. Extensive amounts of myocardial damage would provide a decrease in GLS FS, and EF values. If infarction or ischemia had involved some confronted regions but at under adequate compensation by preserved contraction of either apical or other regions, the resulting EF Simpson value would not have been worse than the decline of FS. In this study, the decline in FS would be equivalent to a decrease in GLS, given that the GLS used in this study was an average GLS of 17 segments. This is in conjunction with the findings of this study which found that there were no meaningful differences in GLS, FS, and $\mathrm{EF}$ values in the group with the infarcted location as well as there were not any difference in the value of all three based on the diagnosis of infarction or noninfarction. This could be explained by Amen where the function of myocardial contractility was strongly determined by the movement of the rotation of the apex to be able to change the shape of the heart space to be smaller each time of contraction, and that study found there was no difference in the regional location of the infarction with the movement of the rotation of the apex. Thus, EF and FS values would be relied on the ability of the apex circumference fibers to cause rotation and reduction of cardiac space. Furthermore, if the area of ischemia and infarction were deep enough to get to radial and circumference fibers, the value of EF and FS would decrease and the decrease will be preceded by a decrease in GLS first. ${ }^{17}$ In addition, in ACS patients, the causes of a decrease GLS can represent many causes from a combination of physiological, metabolic and mechanical causes that affect endocardial fibers. Thus, not all low EF biplanes will have a poorer prognosis based on GLS scores, so it took FS $<25 \%$ to get a lower Simpson EF patient group ( $\mathrm{EF}<50 \%)$ who would have a worse prognosis. In the ACS patient population, there were approximately $10 \%$ of patients found to have $\mathrm{EF}<50 \%$ with no signs of acute heart failure, while of those had signs of acute heart failure there were $35 \%$ patients with $\mathrm{EF}<50 \% .{ }^{18}$ The results of our study could be very useful for the ACS patients in Indonesia who were generally the same, regarding patients spread over the remote areas and were not slightly diagnosed faster or those more likely to justified and ended up being treated conservatively or ACS patients who returned as in a free pain condition but surprisingly found to contributed a high mortality rate. Imaging could help in their high risk stratification of ACS patients and could be the basis of subsequent research of suitable management on a typical ACS subset.

\section{Study Limitation}

Several limitation exist in our study. The relatively small number of MACE / adverse events in this study to perform multivariate analysis on this study. We would also like to state that future studies with larger sample size assessing echocardiographic paramaters studied in this study will have to be done to solidify our findings.

\section{Conclusion}

GLS had a prognostic value in patients with ACS. Left ventricular conventional $\mathrm{M}$-mode FS in addition to Simpson EF were well correlated with GLS as well they can be considered as an alternative in predicting the incident of MACE in patient with ACS.

\section{Acknowledgements}

Cardiac Centre of Adam Malik Hospital Medan, North Sumatra - Indonesia.

\section{References}

1. Theorux P. "Pathogenesis of Stable and Acute Coronary Syndrome.Acute Coronary Syndromes: A Companion to Braunwald's Heart Disease. 2nd. Elsevier Saunders, 2011. 43-58.

2. Darma $S$, et al. Organisation of reperfusion therapy for STEMI in a developing country. Open Heart.2015; 2, e000240, doi:10.1136/ openhrt-2015-000240.

3. Garza MA., Wason, EA., \& Zhang, JQ. Cardiac remodeling and physical training post myocardial infarction. World J Cardiol. 2015; 7(2), 52-64. 
doi:10.4330/wjc.v7.i2.52.

4. Herzog E, Chaudhry F .Echocardiography in Acute Coronary Syndrome,Diagnosis, Treatment, and Prevention.Edited by Herzog E, Chaudhry F.London: Springer; 2010.

5. Mor-Avi V., Lang R M., \& Bandano L. Current and Evolving Echocardiographic Techniques for the Quantitative Evaluation of Cardiac Mechanics: ASE/EAE Consensus Statement on Methodology and Indications Endorsed by the Japanese Society of Echocardiography. J Am Soc Echocardiogr. 2011;24: 277-313.

6. Hu B, Zhou Q, \& Chen J. Prediction For Improvement and Remodeling In First-Onset Myocardial Infarction By Speckle Tracking Echocardiography: Is Global Or Regional Selection Better? Ultrasound in Med.\& Biol. 2017; 1-9. doi:10.1016/j.ultrasmedbio.2017.06.001,

7. Skaarup KG, et al. The impact of cardiovascular risk factors on global longitudinal strain over a decade in the general population: the Copenhagen City Heart Study. The International Journal of Cardiovascular Imaging. 2020, doi: s10554-020-01906-5.

8. Huang SJ, Ting I, Huang AM, Mc.Lean A. Longitudinal wall fractional shortening: an M-mode index based on mitral annular plane systolic excursion (MAPSE) that correlates and predicts left ventricular longitudinal strain (LVLS) in intensive care patients. Critical Care. 2017: 21, 292. doi:10.1186/s13054-017-1876-x

9. Adachi Y, 2016. Left Ventricular Longitudinal Dysfunction Assessed By Longitudinal Fractional. Journal of American Colloege of Cardiology 2016; 67 (13) Supplement, p. 1645.

10. Ibanez B, James S, Agewal S, \& Widimsky P. ESC Guidelines for the management of acute myocardial infarction in patients presenting with ST-segment elevation. European Heart Journal. 2017: 1-66. doi:10.1093/eurheartj/ehx3.

11. Kaufmann D. Global longitudinal straincan predict heart failure exacerbation instable outpatients with schemic left ventricular systolic dysfunction. PLOS ONE,2019: 1-12. doi:10.1371/journal. pone.0225829.

12. Joyce E, Bax, JJ. Association Between Left Ventricular Global LongitudinalStrain and Adverse Left Ventricular Dilatation After ST-
Segment-ElevationMyocardial Infarction. Circ Cardiovasc Imaging. 2014:7,74-81.doi:0.1161/ CIRCIMAGING.113.000982.

13. Park JH. Two-dimensional Echocardiographic Assessment of Myocardial Strain: Important Echocardiographic Parameter Readily Useful in Clinical Field. Korean Circulation Journal. 2019: 10, 9098-931. doi:10.4070/kcj.2019.0200

14. Park K, Chang SA, Kim HK. Normal Ranges and Physiological Changes of Midwall Fractional Shortening in Healthy Korean Population. Korean Circulation Journal. 2010: 40, 587-592. doi:10.4070/kcj.2010.40.11.587

15. Niemeijer MN, Leening M, \& Ven Den Berg ME. Subclinical Abnormalities in Echocardiographic Parameters and Risk of Sudden Cardiac Death in a General Population: The Rotterdam Study. Journal of Cardiac Failure. 2016: 22(1), 17-23. doi:10.1016/j.cardfail.2015.06.007.

16. Luis, S.. Echocardiographic Assessment of Left Ventricular Systolic Function:An Overview of Contemporary Techniques including STE.Mayo Foundation for Medical Education and Journal Proceedings. 2019: 94(1), 125-138.

17. Amen L. Apical Rotation in Patients with STelevation Myocardial Infarction. Menoufia Medical Journal. 2016: 29(2), 412-417. doi:10.4103/11102098.192439.

18. Bermejo RA, et al. Determintants and Prognostic Impact of Heart Failure and Left Ventricular Ejection Fraction in Acute Coronary Syndrome Settings. Revista Espanola de Cardiologia. 2017: 71(10), 820-828. Doi: 10.1016/j.rec.2017.10.030 